\title{
HOMOGENEOUS PREMIUM CALCULATION PRINCIPLES
}

\author{
By AXEL REICH \\ Kölnische Rückversicherungs-Gesellschaft, Cologne
}

\begin{abstract}
A premium calculation principle $\pi$ is called positively homogeneous if $\pi(c X)=$ $c \pi(X)$ for all $c>0$ and all random variables $X$. For all known principles it is shown that this condition is fulfilled if it is satisfied for two specific values of $c$ only, say $c=2$ and $c=3$, and for only all two point random variables $X$. In the case of the Esscher principle one value of $c$ suffices. In short this means that local homogeneity implies global homogeneity. From this it follows that in the case of the zero utility principle or Swiss premium calculation principle, the underlying utility function is of a very specific type.

A very general theorem on premium calculation principles which satisfy a weak continuity condition, is added. Among others the proof uses Kroneckers Theorem on Diophantine Approximations.
\end{abstract}

KEYWORDS

Premium principles, homogeneity, utility functions.

\section{INTRODUCTION}

In actuarial practice one generally uses only three premium calculation principles, namely the expected value principle, the variance principle and the standard deviation principle. Apart from these there are many other principles for determining a premium for a risk: all these are examined in the new textbook by Goovaerts, De Vylder and Haezendonck (1984). A central theme is the analysis of the principles which fulfil some desirable properties such as translation invariance, (sub-)additivity, iterativity, homogeneity etc. For example the expected value principle is always additive and homogeneous, but it is iterative or translation invariant only in the case of a vanishing loading.

If a premium principle is defined by a utility function, then the above mentioned, (so-called) plausible properties are in general very restrictive: The Swiss premium calculation principle e.g. is translation invariant if and only if the corresponding utility function is exponential or linear, and it is positively homogeneous if the utility function $u(x)$ is-up to linear transformations-a power of $x$. Therefore, e.g., the Swiss premium calculation principle is both translation invariant and homogeneous only in the case of a linear utility function. Such an analysis has been performed already for all known principles and all properties mentioned above. If $\pi$ denotes a premium calculation principle, which therefore to any real random variable $X$ assigns a real number $\pi(X)$-the premium of $X$-then in 
all cases it turns out that, e.g., in the case of translation invariance (that means

$$
\pi(X+c)=\pi(X)+c
$$

for all risks $X$ and all real numbers $c$ ), it is sufficient to know equation (1) for all $c \in \mathbb{R}$ and only all $X \in D_{2}\left(D_{2}\right.$ the set of all 2-atomic random variables): If (1) is valid for all $c \in \mathbb{R}, X \in D_{2}$, then automatically (1) is fulfilled also for those $X$ not in $D_{2}$. This reduction to the essence of a property has been worked out for the property of translation invariance by REICH (1984) in a definitive sense: Any principle is already translation invariant (i.e., (1) holds for all $c \in \mathbb{R}$ and all risks $X$ ), if (1) is fulfilled for all $X \in D_{2}$ and two specific values of $c$ only, say $c=1$ and $c=\sqrt{2}$. In case of the Orlicz principle (HAEZENDONCK and GoovaERTS (1982)) a single value of $c$, e.g., $c=1$, suffices. A further reduction is impossible as one can see from the counterexamples in ReICH (1984).

In the case of the property of homogeneity (sometimes also called proportionality)

$$
\pi(c X)=c \pi(X)
$$

(more exactly we will examine positively homogeneous principles, i.e., $c \in \mathbb{R}^{+}$) we will now give a similar analysis of the analogous problems. Equation (2) means for $c=\frac{1}{2}$, say, that the premium of $X$ should be homogeneously divided in two equal parts, if the risk $X$ is split up into two parts in a homogeneous way. The aim of this paper therefore is to give an answer to the question: How little does one really need to know, to have already property (2) in full generality (i.e., for arbitrary risk $X$ and arbitrary $\left.c \in \mathbb{R}^{+}\right)$? Of course, this leads to other conditions than in the case of translation invariance and other principles are now of special interest. A mere corollary from the results (still to be formulated and proved) should be mentioned here: Take for example the Swiss premium calculation principle. If (2) holds for all $X \in D_{2}$ only and for all $c \in\left[\frac{1}{3}, \frac{1}{2}\right]$, then (2) holds automatically for all risks $X$ and all $c \in \mathbb{R}^{+}$. There is therefore no difference in homogeneity as a local or global property. This fact is a trivial consequence of theorem 2.2, which is best possible in the precise sense specified there. Moreover for every known premium calculation principle the following is true $\left(X \in D_{2}\right)$ : If (2) holds in the two special cases $c=\frac{1}{2}$ and $c=\frac{1}{3}$ only, then again (2) is fulfilled for all $c>0$.

From this one can prove that even an extremely weaker assumption than the homogeneity is (with the Orlicz principle as the only exception) very restrictive for all utility principles.

\section{RESULTS AND REMARKS}

Among the known principles the following are in every case (i.e., independent of the choice of the corresponding parameters or utility functions) positively homogeneous: Expected value principle, maximal loss principle, percentile principle, standard deviation principle and Orlicz principle. The variance principle 
on the contrary is certainly not positively homogeneous in the case of a nonvanishing loading (cf. Goovaerts, DE VYlder and HaEzendonck (1984)).

For the remaining cases of the Swiss Premium calculation principle, the zero utility principle (which is indeed a special case of the Swiss premium calculation principle, but has for technical reasons to be treated separately) and the Esscher principle it will now be proved for example: If one has for all $X \in D_{2}$

$$
\pi\left(\frac{1}{2} X\right)=\frac{1}{2} \pi(X) \text { and } \pi\left(\frac{1}{3} X\right)=\frac{1}{3} \pi(X),
$$

then

$$
\pi(c X)=c \pi(X)
$$

holds for all $X$ and all $c \in \mathbb{R}^{+}$, i.e., $\pi$ is positively homogeneous. More generally and more exactly:

\section{A. $\pi=$ zero utility principle}

This principle was introduced by BüHLMANN (1970). One starts with a utility function $u$ with $u^{\prime}(x) \geqslant 0, u^{\prime \prime}(x) \leqslant 0$. For a given risk $X$ the premium $P=\pi(X)$ is determined by

$$
E[u(P-X)]=u(0)
$$

We prove

THEOREM 2.1. For fixed, positive $c_{1}, c_{2} \neq 1$ let $\log c_{1} / \log c_{2}$ not be rational. If for every $X \in D_{2}$

$$
\pi\left(c_{1} X\right)=c_{1} \pi(X) \text { and } \pi\left(c_{2} X\right)=c_{2} \pi(X)
$$

hold, then $u$ is linear. Conversely, if $u$ is linear, then for all $X$ and all $c \in \mathbb{R}^{+}$

$$
\pi(c X)=c \pi(X)
$$

holds, i.e. $\pi$ is positively homogeneous.

REMARK. Theorem 2.1 is best possible in the following sense: For any pair $c_{1}, c_{2} \in \mathbb{R}^{+}\left(c_{1}=c_{2}\right.$ is admissible), which the condition of theorem 2.1 (i.e., $\left.\log c_{1} / \log c_{2} \in \mathbb{Q}\right)$ does not fulfil, there is a non-linear utility function $u$ such that

$$
\pi\left(c_{i} X\right)=c_{i} \pi(X), \quad i=1,2, X \in D_{2},
$$

holds. In this case the zero utility principle certainly is not positively homogeneous.

2B. $\pi=$ Swiss premium calculation principle

This principle was introduced by Bühlmann, Gagliardi, Gerber and STraub (1977): If $z \in[0,1]$ and $u$ is a strictly monotonic, continuous function on $\mathbb{R}$ the 
premium $P=\pi(X)$ for a risk $X$ is given by the equation

$$
E[u(X-z P)]=u((1-z) P) .
$$

In the case $z=1$ and by the substitution $u(x) \rightarrow-u(-x)$ one just gets the zero utility principle. By a different proof than in the case $2 \mathrm{~A}$ one proves for $0 \leqslant z<1$

TheORem 2.2. For fixed, positive $c_{1}, c_{2} \neq 1$ let $\log c_{1} / \log c_{2}$ not be rational. If for every $X \in D_{2}$

$$
\pi\left(c_{1} X\right)=c_{1} \pi(X) \text { and } \pi\left(c_{2} X\right)=c_{2} \pi(X)
$$

hold, then for suitable $\alpha, \beta, \gamma, r \in \mathbb{R}$ (with $\beta \gamma>0, r>0$ )

$$
u(x)= \begin{cases}\alpha+\beta x^{r}, & x \geqslant 0 \\ \alpha-\gamma(-x)^{r}, & x<0 .\end{cases}
$$

Conversely, if $u$ has the form (5), then for all $X$ and all $c \in \mathbb{R}^{+}$

$$
\pi(c X)=c \pi(X)
$$

holds, i.e., $\pi$ is positively homogeneous.

REMARK. Theorem 2.2 is best possible in the following sense: For any pair $c_{1}, c_{2} \in \mathbb{R}^{+}$, which does not fulfil the conditions of theorem 2.2 , there is an admissible utility function $u$, not of the form (5), such that

$$
\pi\left(c_{i} X\right)=c_{i} \pi(X), \quad i=1,2, X \in D_{2}
$$

holds. In this case the Swiss premium calculation principle certainly is not positively homogeneous.

$$
\text { 2C. } \pi=\text { Esscher principle }
$$

This principle was introduced in BüHLMANN (1980) and so named in view of the formal similarity to the Esscher transform: Given $\alpha \geqslant 0$ the premium $P=\pi(X)$ is determined explicitly by the equation

$$
\pi(X)=\frac{E[X \exp (\alpha X)]}{E[\exp (\alpha X)]} .
$$

It is very easy to see (cf. Goovaerts, De Vylder and HAEzendonck (1984)), that the Esscher principle is positively homogeneous only in the case $\alpha=0$, i.e., Esscher premium $=$ net premium. A simple proof will give the following sharp result:

THEOREM 2.3. If for a fixed $c_{0} \neq 1$, a single (non-degenerated) $X_{0} \in D_{2}$ the equation

$$
\pi\left(c_{0} X_{0}\right)=c_{0} \pi\left(X_{0}\right)
$$

holds, then $\alpha=0$. 


\section{D. General premium principles}

The result mentioned in the introduction, namely that the property of positive homogeneity is already fulfilled in the global sense if it is known only locally with respect to the variable $c$, now follows easily: If $\pi$ denotes the zero utility principle, the Swiss premium calculation principle or the Esscher principle, then the theorems above yield at once

COROLlARY 2.4. If for all $X \in D_{2}$ and all $c$ in a given (arbitrarily small) bounded interval in $\mathbb{R}^{+}$one has

$$
\pi(c X)=c \pi(X)
$$

then $\pi(c X)=c \pi(X)$ holds for all $X$ and all $c \in \mathbb{R}^{+}$.

Finally one should pay attention to a very general result, which on the one hand makes the results above more transparent, on the other hand is true for general, possibly still unknown principles: Denote by $\pi$ any premium principle with the very weak and plausible continuity condition, that for every convergent sequence $\left(\gamma_{k}\right) \subset \mathbb{R}^{+}$and every $X \in D_{2}$

$$
\lim _{k \rightarrow \infty} \pi\left(\gamma_{k} X\right)=\pi\left(\lim _{k \rightarrow \infty} \gamma_{k} \cdot X\right)
$$

For such principles one has throughout

THEOREM 2.5. For fixed, positive $c_{1}, c_{2} \neq 1$ let $\log c_{1} / \log c_{2}$ not be rational. If

$$
\pi\left(c_{1} X\right)=c_{1} \pi(X) \quad \text { and } \pi\left(c_{2} X\right)=c_{2} \pi(X), \quad X \in D_{2},
$$

then $\pi(c X)=c \pi(X)$ holds for all $X \in D_{2}$ and even all $c>0$.

As a corollary (because in any interval $I$, however small it may be, there are of course always two numbers $c_{1}, c_{2} \neq 1$ in $I$ such that $\left.\log c_{1} / \log c_{2} \notin \mathbb{Q}\right)$ we note: The (global) property of such premium calculation principles of being positively homogeneous is always a local property in the following sense:

If $\pi(c X)=c \pi(X)$ holds for only all $c \in I$, then automatically also for even all $c \in \mathbb{R}^{+}$.

REMARK. Simple and explicit examples for pairs of numbers $c_{1}, c_{2} \neq 1$, which satisfy $\log c_{1} / \log c_{2} \notin \mathbb{Q}$, are the following:

$$
\begin{array}{ll}
c_{1}=2, & c_{2}=3, \\
c_{1}=\frac{1}{2}, & c_{2}=\frac{1}{3}, \\
c_{1}=1.1, & c_{2}=1.2, \\
c_{1}=2, & c_{2}=\pi, \\
c_{1}=2, & c_{2}=e^{\pi}, \\
c_{1}=e, & c_{2}=e^{\pi}, \\
c_{1}=1.25, & c_{2}=1.1 .
\end{array}
$$


As was pointed out by GERBER (1979), pp. 73-74, the global property of positive homogeneity is not reasonable for practical reasons. From example (vii) e.g., one can deduce by the corollary of theorem 2.5 more precisely that it in the same way again unreasonable to accept the homogeneity property as a local property with respect to $c$ only: If for all $X$

$$
\pi(c X)=c \pi(X)
$$

holds for, say all $c$ between 1 and 1.25 (local property), then automatically by the results above also for all $c>0$ (global property). In accordance with GERBER (1979) the quotient $\pi(c X) / \pi(X)$ should depend not only on $c$ but also on $X$.

\section{PROOFS}

Ad 2A: First of all we consider the zero utility principle $\pi$ with strictly monotonic utility function $u$, such that $u^{\prime}(x) \geqslant 0, u^{\prime \prime}(x) \leqslant 0$. To prove theorem 2.1 we assume $u(0)=0$ without loss of generality, because for a given risk $X$ the premium $P=\pi(X)$ does not change if in (3) $u$ is substituted by $u-u(0)$.

LEMMA 3.1. If for a fixed $c_{0}>0$

$$
\pi\left(c_{0} X\right)=c_{0} \pi(X), \quad X \in D_{2},
$$

then there exists a $\beta_{0}=\beta_{0}\left(c_{0}\right)$ such that

holds for all $x \in \mathbb{R}$.

$$
u\left(c_{0} x\right)=\beta_{0} u(x)
$$

PRoOF. For the present let be $x>0$, and for $a, b \in \mathbb{R}, q \in[0,1]$ let $X=X_{a, b, q} \in D_{2}$ be defined by

$$
\operatorname{pr}(X=a)=1-q, \quad \operatorname{pr}(X=b)=q .
$$

With the abbreviation $P=P(a, b, q)=\pi(X)$ one has by (3)

$$
(1-q) u(P-a)+q u(P-b)=u(0) \text {, }
$$

and by (6)

$$
(1-q) u\left(c_{0}(P-a)\right)+q u\left(c_{0}(P-b)\right)=u(0) .
$$

(7) and (8) yield

$$
\begin{aligned}
& \frac{1-q}{q}=\frac{u(P-b)-u(0)}{u(0)-u(P-a)} \\
& \frac{1-q}{q}=\frac{u\left(c_{0}(P-b)\right)-u(0)}{u(0)-u\left(c_{0}(P-a)\right)}
\end{aligned}
$$

Putting $x^{\prime}=-1, a=2, \gamma=1, b=1-x$ one gets

$$
a=\gamma-x^{\prime}, \quad b=\gamma-x \text { and } \quad b<\gamma<a .
$$


If

$$
q=\frac{u(0)-u\left(x^{\prime}\right)}{u(x)-u\left(x^{\prime}\right)}
$$

then $0<q<1$ in view of the strict monotonicity of $u$. Because of $x=\gamma-b$, $x^{\prime}=\gamma-a$ and by (12) one concludes

$$
(1-q) u(\gamma-a)+q u(\gamma-b)=u(0),
$$

therefore $\gamma=P(a, b, q)$ according to (7). From this, in view of (10), (11) it follows

$$
\frac{1-q}{q}=\frac{u\left(c_{0} x\right)-u(0)}{u(0)-u\left(c_{0} x^{\prime}\right)}
$$

for arbitrary $x>0$. Together with (9) this leads to

$$
\frac{u\left(c_{0} x\right)-u(0)}{u(0)-u\left(c_{0} x^{\prime}\right)}=\frac{u(x)-u(0)}{u(0)-u\left(x^{\prime}\right)}
$$

Therefore

$$
\begin{gathered}
u\left(c_{0} x\right)-u(0)=\frac{u(0)-u\left(c_{0} x^{\prime}\right)}{u(0)-u\left(x^{\prime}\right)}[u(x)-u(0)] \\
u\left(c_{0} x\right)=\frac{u(0)-u\left(c_{0} x^{\prime}\right)}{u(0)-u\left(x^{\prime}\right)} u(x)+u(0)\left[1-\frac{u(0)-u\left(c_{0} x^{\prime}\right)}{u(0)-u\left(x^{\prime}\right)}\right] \\
=\frac{u\left(c_{0} x^{\prime}\right)}{u\left(x^{\prime}\right)} u(x)
\end{gathered}
$$

for all $x>0$, respecting the normalization $u(0)=0$. With $\beta_{0}=u\left(c_{0} x^{\prime}\right) / u\left(x^{\prime}\right)$ this is the assertion for $x>0$.

In the case $x \leqslant 0$ one proves in an analogous way the existence of a real number $\gamma_{0}$ such that

$$
u\left(c_{0} x\right)=\gamma_{0} u(x)
$$

holds for all $x \leqslant 0$. Now certainly $\beta_{0}=\gamma_{0}$ (this is exactly the statement of lemma 3.1 ), because with regard to (9) and $u(0)=0$ one has

$$
\frac{1-q}{q}=-\frac{u(P-b)}{u(P-a)} \text {. }
$$

Correspondingly by (10)

$$
\frac{1-q}{q}=-\frac{u\left(c_{0}(P-b)\right)}{u\left(c_{0}(P-a)\right)}=-\frac{\beta_{0}}{\gamma_{0}} \cdot \frac{u(P-b)}{u(P-a)}
$$

if $b<P<a$, which is true in view of (11) and $P=\gamma$. Comparing (15) and (16) one has $\beta_{0}=\gamma_{0}$. 
Proof of Theorem 2.1. Under the assumptions of theorem 2.1 there are by lemma 3.1 real numbers $\beta_{1}, \beta_{2}$ such that

$$
u\left(c_{1} x\right)=\beta_{1} u(x) \text { and } u\left(c_{2} x\right)=\beta_{2} u(x), \quad x \in \mathbb{R} .
$$

Successive application of these relations gives for $x=1$

$$
u\left(c_{1}^{n} c_{2}^{m}\right)=\beta_{1}^{n} \beta_{2}^{m} u(1), \quad n, m \in \mathbb{Z} .
$$

Together with (17) it follows for $x \in \mathbb{R}, n, m \in \mathbb{Z}$

$$
u\left(c_{1}^{n} c_{2}^{m} x\right)=u\left(c_{1}^{n} c_{2}^{m}\right) u(x) / u(1) .
$$

By assumption one has $\log c_{1} / \log c_{2} \notin \mathbb{Q}$, therefore according to Kronecker's approximation theorem (cf. REICH (1984), Appendix) the set

$$
\left\{k \log c_{1}+l \log c_{2}: k, l \in \mathbb{Z}\right\}
$$

is dense in $\mathbb{R}$. From this it follows at once that for every given number $y>0$ there are two sequences $k(n), l(n) \in \mathbb{Z}$ such that

$$
y=\lim _{n \rightarrow \infty} c_{1}^{k(n)} c_{2}^{l(n)}
$$

By (18) and the continuity of $u$ one concludes

$$
u(y x)=u(y) u(x) / u(1) .
$$

for arbitrary $y>0, x \in \mathbb{R}$. The only continuous solution of this functional equation are

$$
u(x)=u(1) x^{r}, \quad x>0,
$$

with some $r \in \mathbb{R}$, as is well known. Because $u$ is strictly increasing, (21) holds for all $x>0$ with suitable $r>0$. Moreover, if $x<0$ then it follows from (20)

$$
u(x)=u(-1)(-x)^{r},
$$

so indeed there are numbers $\beta>0, \gamma<0$ such that

$$
u(x)=\left\{\begin{array}{ll}
\beta x^{r}, & x \geqslant 0 \\
\gamma(-x)^{r}, & x<0
\end{array} .\right.
$$

In the case $r=1$ one has certainly $\beta=-\gamma$ for continuous $u^{\prime}$, therefore $u$ is linear. The case $r \geqslant 2$ is impossible in view of $u^{\prime \prime}(x) \leqslant 0$, the case $0<r<2, r \neq 1$, is impossible according to the existence of $u^{\prime \prime}(0)$. Because of the assumed normalization of $u$ theorem 2.1 is proved.

The remark after theorem 2.1 can be easily proved.

$A d 2 B:$

Proof of Theorem 2.2 (Swiss premium calculation principle). Let be $z<1$ and without any restriction of generality let $u$ be strictly increasing. Assume as 
in theorem 2.2 that two numbers $c_{1}, c_{2} \neq 1$ are given and that $\pi\left(c_{i} X\right)=c_{i} \pi(X)$ holds for $i=1,2$. According to (4) one has more precisely

$$
E\left[u\left(c_{i} X-z c_{i} P\right)\right]=u\left((1-z) c_{i} P\right), \quad X \in D_{2}, \quad i=1,2 .
$$

Defining $g_{i}(x)=u\left(c_{i} x\right)$ equation (22) gives

$$
E\left[g_{i}(X-z P)\right]=g_{i}((1-z) P) \text {. }
$$

By Goovaerts, de Vylder and Haezendonck (1984), theorem 2, p. 72, there exist real numbers $\alpha_{i}, \beta_{i}, i=1,2$, such that

$$
u\left(c_{i} x\right)=\alpha_{i}+\beta_{i} u(x), \quad x \in \mathbb{R} .
$$

Without any restriction one can assume $u$ to be normalized, especially $u(0)=0$. Then, of course, $\alpha_{i}=0$ and

$$
u\left(c_{i} x\right)=\beta_{i} u(x), \quad x \in \mathbb{R} .
$$

From this it follows immediately for arbitrary $n, m \in \mathbb{Z}, x \in \mathbb{R}$

$$
u\left(c_{1}^{n} c_{2}^{m} x\right)=\beta_{1}^{n} \beta_{2}^{m} u(x)=u\left(c_{1}^{n} c_{2}^{m}\right) u(x) / u(1) .
$$

The condition $\log c_{1} / \log c_{2} \notin \mathbb{Q}$ leads via Kronecker's approximation theorem and the continuity of $u$ to

$$
u(y x)=u(y) u(x) / u(1)
$$

for all $y>0, x \in \mathbb{R}$.

In the case $x \geqslant 0$ one introduces $u_{1}(x)=u(x) / u(1)$ and gets by (26)

$$
u_{1}(y x)=u_{1}(y) u_{1}(x), \quad x, y>0 .
$$

As is well known for continuous $u_{1}$ it follows that $u_{1}$ is monomial, therefore $u$ too:

with suitable $r>0$.

$$
u(x)=u(1) x^{r}, \quad x \geqslant 0,
$$

In the case $x<0$ one defines $z=-x$ and $u_{2}(z)=-u(z)$. By (26)

$$
u_{2}(y z)=u_{2}(y) u_{2}(z) / u_{2}(1) \text {, }
$$

therefore it follows in a similar way that $u_{2}$ is a monomial. This means

$$
u(x)=-u(-1)(-x)^{s}, \quad x<0,
$$

for suitable $s>0$. In view of (26) $r=s$ holds, so the first part of theorem 2.2 is proved. The second part is trivial.

The remark after theorem 2.2 is easily proved and the proof is omitted.

Ad 2C:

Proof of Theorem 2.3 (Esscher principle). Let $X_{0} \in D_{2}$ not be degenerated, say

$$
\operatorname{pr}\left(X_{0}=a\right)=1-q, \quad \operatorname{pr}\left(X_{0}=b\right)=q
$$


for some $a, b \in \mathbb{R}, a \neq b, q \in(0,1)$. If for a fixed $c_{0} \neq 1$

$$
\pi\left(c_{0} X_{0}\right)=c_{0} \pi\left(X_{0}\right)
$$

is true, then

$$
\frac{c_{0} a(1-q) \exp \left(\alpha c_{0} a\right)+c_{0} b q \exp \left(\alpha c_{0} b\right)}{(1-q) \exp \left(\alpha c_{0} a\right)+q \exp \left(\alpha c_{0} b\right)}=c_{0} \frac{a(1-q) \exp (\alpha a)+b q \exp (\alpha b)}{(1-q) \exp (\alpha a)+q \exp (\alpha b)} \text {. }
$$

Multiplication yields

$$
(a-b) \exp \left[\alpha\left(c_{0} a+b\right)\right]=(a-b) \exp \left[\alpha\left(c_{0} b+a\right)\right]
$$

therefore in view of $a \neq b$

$$
\exp \left[\alpha\left(c_{0} a+b\right)\right]=\exp \left[\alpha\left(c_{0} b+a\right)\right]
$$

Assume $\alpha>0$, then $c_{0} a+b=c_{0} b+a$, therefore $a=b$ according to $c_{0} \neq 1$. This is a contradiction, so necessarily $\alpha=0$.

Ad 2D:

If $I=(a, b), 0 \leqslant a<b \leqslant \infty$, is any interval in $\mathbb{R}^{+}$, then by trivial arguments there are two numbers $c_{1}, c_{2} \in I$ such that $\log c_{1} / \log c_{2} \notin \mathbb{Q}$. From this it is clear that Corollary 2.4 follows from the preceding Theorems.

Proof of Theorem 2.5. By induction one immediately proves for $n, m \in \mathbb{N}$

$$
\pi\left(c_{1}^{n} X\right)=c_{1}^{n} \pi(X), \quad \pi\left(c_{2}^{m} X\right)=c_{2}^{m} \pi(X) .
$$

Because of

$$
\pi(X)=\pi\left(c_{i} \cdot \frac{1}{c_{i}} X\right)=c_{i} \pi\left(\frac{1}{c_{i}} X\right), \quad i=1,2,
$$

equation (27) even holds for $n, m \in \mathbb{Z}$. Then one has

$$
\pi\left(c_{1}^{n} c_{2}^{m} X\right)=c_{1}^{n} \pi\left(c_{2}^{m} X\right)=c_{1}^{n} c_{2}^{m} \pi(X)
$$

Given an arbitrary $c>0$ there are according to Kronecker's approximation theorem (cf. REICH (1984), Appendix) two sequences $n(k), m(k) \in \mathbb{Z}$ such that

$$
\gamma_{k}=c_{1}^{n(k)} c_{2}^{m(k)} \rightarrow c .
$$

The continuity condition for $\pi$ and (28) yields

$$
\pi(c X)=\pi\left(\lim _{k \rightarrow \infty} \gamma_{k} \cdot X\right)=\lim _{k \rightarrow \infty} \pi\left(\gamma_{k} X\right)=\lim _{k \rightarrow \infty} \gamma_{k} \cdot \pi(X)=c \pi(X),
$$

therefore the assertion. 


\section{REFERENCES}

BühlmanN, H. (1970). Mathematical Methods in Risk Theory. Springer: Berlin-Heidelberg-New York.

Bühlmann, H., B. Gagliardi, H. U. Gerber and E. Straub (1977). Some Inequalities for Stop-Loss Premiums. ASTIN Bulletin 9, 75-83.

Bühlmann, H. (1980) An Economic Premium Principle. ASTIN Bulletin 11, 52-60.

GERBER, H. U. (1979) An Introduction to Mathematical Risk Theory. Huebner Foundation: Philadelphia.

Goovaerts, M. J., F. De Vylder and J. HAezendonck (1984) Insurance Premiums. NorthHolland: Amsterdam.

Haezendonck, J. and Goovaerts, M. J. (1982) A new Premium Calculation Principle based on Orlicz Norms. Insurance: Mathematics and Economics 1, 41-53.

ReICH, A. (1984). Premium Principles and Translation Invariance. Insurance: Mathematics and Economics 3, 57-66. 